\title{
Isolation and Identification of Urinary Tract Infectious Bacteria and Exploring their Anti-drug Potential against Some Common Antibiotics
}

Karzan Mohammed K, Faeza Burhan $\mathbf{O}$ and Shahida Nooruldeen $\mathbf{Y}$

Department of biology, Soran University, Iraq

\begin{abstract}
Urinary tract infection caused by bacteria leads to inflammation and over growth of uropathogens and prevalence of infection for both genders, but women is more vulnerable especially at the sexually active ages. Nine isolates from sixteen patients were microscopically tested, characterized, identified using different media and biochemical tests. The highest rate of isolated bacteria were Escherichia coli and Staphylococcus aureus (23.52\%), followed by Staphylococcus saprophyticus, Entrococcus faecalis (17.64\% and 8.82), respectively and Entrobacter aerogenes, Klebsiella pneumonia, Proteus vulgaris and Pseudomonas aeruginosa were (5.88\%), only $2.94 \%$ of bacteria was detected as Proteus miralilis. Effect of different antibiotics was reported, maximum effect showed by Gentamycin and Chloramphenicol (80\% and $70 \%$ ), respectively. Contrastingly, levofloxacin $50 \%$, Amikacin and Nitrofurantoin $40 \%$, Ceftriaxone and Amoxicillin $30 \%$, Cefixime $10 \%$. In conclusion, unsuitable medication prior to urine culturing causes to increase prevalence of gram positive bacteria as much as gram negatives and developing multidrug resistance.
\end{abstract}

Keywords: Urinary tract infection; Uropathogenic bacteria; Antibiotic; Resistance; Agar well diffusion

\section{Introduction}

Urinary Tract Infection is classified as the most common and occurring nosocomial bacterial infection in human populations around the world [1-3]. UTI is a condition caused by pathogenic invasion of the epithelium, which lines the urinary tract from the minor calyx to prostatic urethra. The proliferation of bacteria in the urothelium can be asymptomatic or symptomatic, which causes inflammatory response and symptomatic case characterized by a wide range of symptoms including, fever, lethargy, anorexia and vomiting [4-9]. However, both genders are susceptible to this type of infection, but women are more, as their reproductive anatomy and physiology are more sensitive. Half of all women by 32 years age had experienced at least an infection history $[7,10]$.

Normally, urinary tract urine mostly dominated by E. coli $75 \%$ $80 \%$, followed by S. saprophyticus 10-15\% [11-15]. While, Anatomy or physiological factors cause abnormality of urinary tract and lead to localize infectious bacteria, such as different species of Klebsiella, Proteus, Enterobacter, Enterococcus, Staphylococcus and Pseudomonas aeruginosa. Those bacteria are more common in most of the cases, and infrequently cause to uncomplicated cystitis and pyelonephritis $[11,12,16]$. Furthermore, pathogenesis of Urinary tract is more complicated and influenced by other factors, such as vaginal ecosystem especially Lactobacillus spp., intestinal population, genetic and behavioral factors, virulence properties of uropathogens and host defense factors [17-19]. The presence of factors will increase opportunity for uropathogens to colonize and invade urothelium [20-22].

Treating urinary tract must be done by the correct diagnosis of symptomatic patients and adequate antimicrobial therapy is taken, with correct dose and route of administration. Intestinal mucosal protection as a mechanical barrier proposed to reduce uropathogen adherence to urothelium. Bacterial proliferation and decrease of the load of UPEC in the intestinal lumen and in the fecal material as non-antibiotic approach therapy $[20,23]$.

As UTI is one of the most prevalent problem among people of all ages and both genders and because the infection mostly associated with gram negative uropathogenic E. coli (UPEC) [13]. For these reasons, the antibiotics which are prescribed by physician mostly effective against gram negative uropathogen. Actually, in most cases of UTI associated with bacterial infection many species of gram positive bacterium are detected as infectious agent. In order to maintaining the level of bacterial normal flora at the appropriate rate and prevention from growth of antibacterial resistance pathogen due to the usage of unnecessary and impracticable antibiotic [24].

Our aim is to isolate, characterize and identify the uropathogens in our endemic region and determine the susceptibility of each isolated organism against several selected antibiotics, which are commonly consumed by patients against bacterial infection in urinary tract. The mean point of this study is to perform the consciousness for using the urine culture sensitivity for each patient and detecting the potential of antibiotics qualitatively and quantitatively prior to use any course of antibiotic or anti-inflammation therapy.

\section{Materials and Methods}

\section{Sample collection}

Patients were asked to clean their external genitalia with disinfectant and collect midstream urine in sterilized cap. Samples were kept in ice bag and directly transported to microbiological laboratory.

\section{Physical examination}

$(\mathrm{pH}$, color, volume, appearance) parameters of collected urine specimens were analyzed.

\section{Urine culturing}

Urine samples were cultured on Nutrient agar, blood agar and

*Corresponding author: Karzan Mohammed K, Department of Biology, Soran University, Iraq; Tel: 964663506666; E-mail: karzan.khalid@bio.soran.edu.krd

Received October 04, 2017; Accepted November 22, 2017; Published November 29, 2017

Citation: Karzan Mohammed K, Faeza Burhan O, Shahida Nooruldeen Y (2017) Isolation and Identification of Urinary Tract Infectious Bacteria and Exploring their Anti-drug Potential against Some Common Antibiotics. J Microb Biochem Technol 9: 285-289. doi: 10.4172/1948-5948.1000379

Copyright: () 2017 Karzan Mohammed K, et al. This is an open-access article distributed under the terms of the Creative Commons Attribution License, which permits unrestricted use, distribution, and reproduction in any medium, provided the original author and source are credited. 
MacConkey agar medium and incubated over night at $37^{\circ} \mathrm{C}$. Significant growth was evaluated as $\geq 10^{5}$ colony-forming units $\mathrm{CFU} / \mathrm{mL}$ of midstream urine.

\section{Characterization and identification}

Culture characteristics: Each of the color, size, elevation, margins and texture of colonies were screened. The morphological different colonies on MacConkey agar, nutrient agar and blood agar were subcultured into nutrient agar medium, in order to purify the isolated bacteria from each patient urine specimen.

Microscopically examination: Pure isolates were examined microscopically, on the base of their cell wall composition and presence of capsule.

Microbiological analysis: According to gram staining technique, isolates were cultured on numerous selective and differential media to find out their color, colony morphology and ability of fermentation.

\section{Prepared media}

Several types of media were used to grow up as well as characterize obtained isolated, such as; Nutrient agar, Mac Conky agar, Blood agar, Pepton water, Simmon citrate agar, EMB, MSA, MHA, Casein agar, TSI, Semi-solid nutrient agar.

All the above media were autoclaved at $121^{\circ} \mathrm{C}$ for $15 \mathrm{~min}$.

\section{Biochemical test}

Selected colonies were identified and differentiate according to the culture characteristics, microscopical examination and microbiological analysis were tested biochemically for further confirmation of isolated bacteria, such as; TSI, Catalase, Oxidase, Indole production test, MR test, VP test, Citrate utilization test, Motility test and Casein hydrolysis.

\section{Antimicrobial sensitivity testing (Kirby-Bauer method)}

The susceptibility of isolates to antibiotics were demonstrated by using nine specific antibiotics, including prescribed antibiotics that have been given by physician (Gentamycin, Penicillin, Amikacin, Chloramphenicol, Cefixime, Ceftriaxone, Amoxicillin, Nitrofurantoin, Levofloxacin).

Isolates were inoculated in peptone water and incubated in $37^{\circ} \mathrm{C}$, 18-24 h. Next, they were re-cultured in broth and their turbidity compared to $0.5 \mathrm{Mcfarland}$ standard solutions. More ever, new cultures were plated on Mueller-Hinton agar by swabbing. After drying for about 5-10 min, Plates were incubated for about $10-15 \mathrm{~min}$ at $37^{\circ} \mathrm{C}$. Furthermore, interested antibiotic discs were adjusted on cultured plates using sterile forceps and incubated as inverted for $24 \mathrm{~h}$ at $37^{\circ} \mathrm{C}$.

\section{Results and Discussion}

Urine sample of sixteen patients were collected from Ashti Hospital, Soran city, Kurdistan. Microscopically, they were examined and physical properties recorded as a primitive stage of detecting inflammation, due to the presence of RBCs, crystal, epithelial cells, color, reaction and appearance as shown in Table 1. Samples were cultured and thirty four different bacteria obtained from nine different species. For this reason, all the colony characteristics were recorded to more recognize isolates and differentiation between them on various types of media (Table 2 ), because monitoring bacteria on media is an initial achievement to identify bacteria on the basis of colony appearance [25]. Furthermore, among nine isolated bacteria only three were gram positive and cocci shape, the remained gram negative and bacilli shape.

\begin{tabular}{|c|c|c|c|c|c|}
\hline Patient code & Age & Gender & Color & Appearance & pH \\
\hline $\mathbf{1}$ & 25 & Female & white & Clear & Acidic \\
\hline $\mathbf{2}$ & 18 & Female & $\begin{array}{c}\text { Pale } \\
\text { yellow }\end{array}$ & Turbid & Acidic \\
\hline $\mathbf{3}$ & 32 & Female & White & Clear & Acidic \\
\hline $\mathbf{4}$ & 22 & Male & Yellow & Clear & Acidic \\
\hline $\mathbf{5}$ & 30 & Female & Yellow & Clear & Acidic \\
\hline $\mathbf{6}$ & 35 & Male & Yellow & Turbid & Acidic \\
\hline $\mathbf{7}$ & 32 & Female & Pale & Clear & Acidic \\
\hline $\mathbf{8}$ & 40 & Female & Red & Turbid & Acidic \\
\hline $\mathbf{9}$ & 65 & Male & Yellow & Turbid & Acidic \\
\hline $\mathbf{1 0}$ & 27 & Male & Yellow & Clear & Acidic \\
\hline $\mathbf{1 1}$ & 38 & Male & Pale & Clear & Acidic \\
\hline $\mathbf{1 2}$ & 25 & Female & Yellow & Turbid & Acidic \\
\hline $\mathbf{1 3}$ & 27 & Female & White & Clear & Acidic \\
\hline $\mathbf{1 4}$ & 48 & Male & Yellow & Clear & Acidic \\
\hline $\mathbf{1 5}$ & 21 & Female & Yellow & Clear & Acidic \\
\hline $\mathbf{1 6}$ & 23 & Female & Yellow & Turbid & Acidic \\
\hline & Table & Phal & & \\
\hline
\end{tabular}

Table 1: Physical parameters of urine samples.

\begin{tabular}{|c|c|c|}
\hline Patient No. & Isolates & $\begin{array}{c}\text { Number of } \\
\text { uropathogen present }\end{array}$ \\
\hline 1 & $\begin{array}{c}\text { Escherichia coli } \\
\text { Staphylococcus saprophyticus }\end{array}$ & 2 \\
\hline 2 & $\begin{array}{c}\text { Escherichia coli } \\
\text { Staphylococcus aureus }\end{array}$ & 2 \\
\hline 3 & Escherichia coli & 1 \\
\hline 4 & $\begin{array}{l}\text { Staphylococcus saprophyticus } \\
\text { Staphylococcus aureus }\end{array}$ & 2 \\
\hline 5 & $\begin{array}{l}\text { Klebsiella pneumonia } \\
\text { Enterobacter aerogenes } \\
\text { Staphylococcus aureus } \\
\text { Staphylococcus saprophyticus } \\
\text { Proteus mirabilis }\end{array}$ & 5 \\
\hline 6 & Staphylococcus aureus & 1 \\
\hline 7 & Enterobacter aerogenes & 1 \\
\hline 8 & $\begin{array}{c}\text { Staphylococcus aureus } \\
\text { Staphylococcus saprophyticus } \\
\text { Proteus vulgaris }\end{array}$ & 3 \\
\hline 9 & Staphylococcus saprophyticus & 1 \\
\hline 10 & $\begin{array}{c}\text { Staphylococcus aureus } \\
\text { Pseudomonas aeruginosa } \\
\text { Escherichia coli }\end{array}$ & 3 \\
\hline 11 & Enterococcus faecalis & 1 \\
\hline 12 & $\begin{array}{c}\text { Escherichia coli } \\
\text { Staphylococcus aureus }\end{array}$ & 2 \\
\hline 13 & $\begin{array}{c}\text { Pseudomonas aeruginosa } \\
\text { Kliebsiella pneumonia } \\
\text { Escherichia coli } \\
\text { Proteus mirabilis }\end{array}$ & 4 \\
\hline 14 & $\begin{array}{l}\text { Staphylococcus aureus } \\
\text { Escherichia coli } \\
\text { Enterococcus faecalis }\end{array}$ & 3 \\
\hline 15 & Staphylococcus saprophyticus & 1 \\
\hline 16 & $\begin{array}{l}\text { Staphylococcus aureus } \\
\text { Enterococcus faecalis } \\
\text { Escherichia coli }\end{array}$ & 2 \\
\hline
\end{tabular}

Table 2: Isolated bacteria in urine sample.

Various types of specific media were prepared to cultivate and characterize isolates, such as; Blood agar, Nutrient agar for all isolates. EMB and MacConky agar for gram negative bacteria, while MSA used as a selective and differential medium of $S$. aureus (Table 3 ). 
Citation: Karzan Mohammed K, Faeza Burhan O and Shahida Nooruldeen Y (2017) Isolation and Identification of Urinary Tract Infectious Bacteria and Exploring their Anti-drug Potential against Some Common Antibiotics. J Microb Biochem Technol 9: 285-289. doi: 10.4172/1948-5948.1000379

Then, several biochemical tests were performed for gram negatives, such as IMViC test and TSI as the most special tests to identify them. Regarding present or absent of catalase and oxidase enzyme, there were only E. faecalis (-) and P. aeroginosa (+) was respectively. Motility test was applied to all isolated bacteria, all gram negatives motile excluding $K$. pneumonia, while no gram positive motile. Casein was used to differentiate
Enterococcus faecalis and E. coli from other gram negatives, which indicated their ability to casein hydrolysis (Table 4). S. saprophyticus was resistant to Novobiocin, which is the only promised antibiotic that allows all species of Staphylococcus to grow except S. saprophyticus [26].

As a result, nine species were identified from sixteen patients, the

\begin{tabular}{|c|c|c|}
\hline Media & Uropathogen & Colony morphology \\
\hline \multirow{6}{*}{ MacConkey agar } & Escherichia coli & Lactose-fermenting 'shiny, rose pink \\
\hline & Klebsiella pneumonia & Pink, colony, large 'glistening and mucoid \\
\hline & Pseudomonas aeruginosa & Flat, blue-green diffusible pigment, feathery \\
\hline & Proteus mirabilis & Lactose-non fermenting 'colourless colony \\
\hline & Proteus vulgaris & Lactose-non fermenting 'colorless colony \\
\hline & Enterobacter aerogenes & Lactose fermenter, light pink, unbonate small \\
\hline \multirow{5}{*}{ Eosin methylene blue agar } & Escherichia coli & Green metallic sheen colonies \\
\hline & Klebsiella pneumonia & Large, pink to purple colonies \\
\hline & Pseudomonas aeruginosa & Diffusible, purple 'rough \\
\hline & Enterobacter aerogenes & Pink, colony without metalic green sheen \\
\hline & Proteus mirabilis & Irregular spreadable, small, colorless colony \\
\hline \multirow{3}{*}{ Mannitol salt agar } & Proteus vulgaris & Small color less colony \\
\hline & Staphylococcus aureus & Yellow colony, mannitol fermenter \\
\hline & Staphylococcus saprophyticus & Fermenter mannitol non-color less to white \\
\hline \multirow{10}{*}{ Blood agar } & Enterococcus faecalis & White color weak mannitol fermenter \\
\hline & Escherichia coli & Shiny, opaque, creamy .B-hemolytic \\
\hline & Klebsiella pneumonia & y-hemolysis, hemolysis, grey color \\
\hline & Pseudomonas aeruginosa & B-hemolysis Grayishcolonies, oblique lighting \\
\hline & Enterobacter aerogenes & White circle colony of Y-hemolysis \\
\hline & Proteus mirabilis & Swarming spread film growth white to grey \\
\hline & Proteus vulgaris & Non swarming grey colony \\
\hline & Staphylococcus aureus & Small white to creamy, B-hemolysis \\
\hline & Staphylococcus saprophyticus & White circle colony of $\mathrm{Y}$-hemolysis \\
\hline & Enterococcus faecalis & Small smooth colony, Y-hemolysis \\
\hline \multirow{9}{*}{ Nutrient agar } & Escherichia coli & Circle, convex, small colonies \\
\hline & Klebsiella pneumonia & Circle, convex, small mucoid colonies \\
\hline & Pseudomonas aeruginosa & Large, opaque, produce diffusible pigment \\
\hline & Enterobacter aerogenes & Small, creamy convex, smooth \\
\hline & Proteus mirabilis & Spreadable, swarming white to creamy colony \\
\hline & Proteus vulgaris & Non swarming circular smooth entire, opaque \\
\hline & Staphylococcus saprophyticus & White to pink circular*convex \\
\hline & Enterococcus faecalis & Small, entire, circular, opaque, white convex \\
\hline & Staphylococcus aureus & Entire, white pinhead (punctiform)convex \\
\hline
\end{tabular}

Table 3: Isolated bacteria on different media.

\begin{tabular}{|c|c|c|c|c|c|c|c|c|c|}
\hline BIOCHEMICAL TESTS & E. faecalis & E. coli & K. pneumonia & S. aureus & P. vulgaris & S. saprophyticus & P. mirabilis & $P$. aeruginosa & E. aerogenes \\
\hline Gram stain & + & - & - & + & - & + & - & - & - \\
\hline Shape & Cocci & Rod & Rod & Cocci & Rod & Cocci & Rod & Rod & Rod \\
\hline Capsule stain & - & - & + & - & - & - & - & - & - \\
\hline TSI & A/A & $\mathrm{A} / \mathrm{A}, \mathrm{G}$ & $A / A, G$ & $\mathrm{~A} / \mathrm{A}$ & $\mathrm{A} / \mathrm{A}, \mathrm{G}, \mathrm{H}_{2} \mathrm{~S}$ & $\mathrm{~A} / \mathrm{A}$ & $\mathrm{K} / \mathrm{A}, \mathrm{G}, \mathrm{H}_{2} \mathrm{~S}$ & $\mathrm{~K} / \mathrm{K}$ & $K / A, G$ \\
\hline Catalase & - & + & + & + & + & + & + & + & + \\
\hline Oxidase & - & - & - & - & - & - & - & + & - \\
\hline Indole production test & & + & - & & + & & - & - & - \\
\hline MR test & & + & - & & + & & + & - & - \\
\hline VP test & & - & - & & - & & - & - & + \\
\hline Citrate utilization test & & - & + & & - & & + & + & + \\
\hline Motility test & - & + & - & - & + & - & + & + & - \\
\hline Casein hydrolysis & + & + & - & - & + & - & - & - & - \\
\hline
\end{tabular}

Table 4: Biochemical test for isolated bacteria. 


\begin{tabular}{|c|c|c|c|c|c|c|c|c|c|c|c|c|c|c|c|c|c|c|c|}
\hline \multirow{2}{*}{ Antibiotic (mcg) } & \multicolumn{2}{|c|}{ E. coli } & \multicolumn{2}{|c|}{ K. pneumonia } & \multicolumn{2}{|c|}{ P. aeruginosa } & \multicolumn{2}{|c|}{ E. aerugenes } & \multicolumn{2}{|c|}{ P. mirabilis } & \multicolumn{2}{|c|}{ P. vulgaris } & \multicolumn{2}{|c|}{ E. faecalis } & \multicolumn{2}{|c|}{ S. aureus } & \multicolumn{2}{|c|}{ S. saprophyticus } & \multirow{2}{*}{\begin{tabular}{|c|}
$\%$ OF \\
sensitivity
\end{tabular}} \\
\hline & S/R & $\mathrm{mm}$ & $\mathbf{S} / \mathbf{R}$ & $\mathbf{m m}$ & S/R & $\mathrm{mm}$ & S/R & $\mathbf{m m}$ & S/R & mm & S/R & $\mathbf{m m}$ & S/R & $\mathbf{m m}$ & S/R & $\mathbf{m m}$ & S/R & $\mathrm{mm}$ & \\
\hline Levofloxacin (L) (5 mcg/10 mL) & S & 24 & $\mathrm{R}$ & - & $\mathrm{S}$ & 14 & $\mathrm{R}$ & - & S & 21 & $\mathrm{R}$ & - & S & 28 & $\mathrm{~S}$ & 18 & $\mathrm{R}$ & - & 50 \\
\hline Amoxicillin (AMX) (25) & S & 22 & $\mathrm{R}$ & - & $\mathrm{S}$ & 23 & $\mathrm{R}$ & - & $\mathrm{R}$ & - & $\mathrm{R}$ & - & S & 17 & $\mathrm{R}$ & - & $\mathrm{R}$ & - & 30 \\
\hline Gentamycin (G) (8000 mcg/2 mL) & $S$ & 25 & $\mathrm{~S}$ & 27 & $\mathrm{~S}$ & 23 & $\mathrm{~S}$ & 26 & $\mathrm{~S}$ & 27 & $\mathrm{R}$ & - & $S$ & 26 & $\mathrm{~S}$ & 23 & $\mathrm{~S}$ & 17 & 80 \\
\hline chloramphenicol 30 & S & 25 & $\mathrm{~m}$ & 13 & $\mathrm{~S}$ & 12 & $\mathrm{R}$ & - & $\mathrm{S}$ & 16 & S & 20 & S & 25 & $\mathrm{~S}$ & 25 & $\mathrm{~S}$ & 28 & 70 \\
\hline Amikacin (AK) (10) & $\mathrm{S}$ & 17 & $\mathrm{R}$ & 13 & $\mathrm{~S}$ & 14 & $\mathrm{R}$ & 13 & $\mathrm{R}$ & 13 & S & 19 & S & 20 & $\mathrm{R}$ & 14 & $\mathrm{R}$ & 10 & 40 \\
\hline Nitrofurantoin $(\mathrm{N})(50 \mathrm{mcg} / \mathrm{mL})$ & $S$ & 23 & $S$ & 18 & $\mathrm{~S}$ & 11 & $\mathrm{R}$ & - & $\mathrm{R}$ & - & $\mathrm{R}$ & - & $S$ & 15 & M & 15 & $\mathrm{R}$ & - & 40 \\
\hline cefixime (CFM) (5) & $\mathrm{R}$ & 14 & $\mathrm{R}$ & - & $\mathrm{R}$ & - & $\mathrm{R}$ & - & $\mathrm{R}$ & - & $\mathrm{R}$ & - & $\mathrm{R}$ & - & $\mathrm{S}$ & 14 & $\mathrm{R}$ & - & 10 \\
\hline Ceftriaxone (CRO) (30 mcg) & S & 26 & $\mathrm{R}$ & - & $\mathrm{S}$ & 23 & $\mathrm{R}$ & - & $\mathrm{R}$ & 11 & $\mathrm{R}$ & - & $\mathrm{R}$ & 20 & $\mathrm{~S}$ & 27 & $\mathrm{R}$ & - & 30 \\
\hline
\end{tabular}

S: Sensitive; R: Resistance

Table 5: Antibiotic sensitivity test Kerby-Bauer method.

highest rate of bacteria were E. coli and S. aureus (23.52\%), followed by S. saprophyticus, Enterococcus faeculis ( $17.64 \%$ and 8.82$)$, respectively. E. aeroginosa, K. pneumonia, P. vulgaris and P. aeroginosa (5.88\%), and only $2.94 \%$ of bacteria was detected to be P. miralilis. According to the highest rate of infections due to E. coli and maximum number of isolates is E. coli. However, S. aureus can be seen in urinary tract but less than $E$. coli, but in this study this rate was as much as E. coli high, may be due to contamination of urine during urinate, which is caused by bacteria on skin, or no use of disinfectants to remove microorganisms on skin in this area $[19,27]$. Sensitivity and resistance of isolates for various antibiotics were reported in Table 5.

Gentamycin was $80 \%$ effective, followed by Chloramphenicol $70 \%$ and Levoxacin 50\%, Amikacin, Nitrofurantoin $40 \%$ and both Ceftriaxone and Amoxicillin 30\%, less effectiveness showed by Cefixime $10 \%$. The obtained data of this study were represented that most of the people who are infected by UTI, belong to the age group of 25-35 years $[28,29]$.

\section{Conclusion}

Nine isolate of bacteria were identified from sixteen patients. Their sensitivity to nine antibiotics was performed and the activity of antibiotics for inhibiting bacterial growth was at different levels, According to their ability. We concluded that people at age group of 2535 highly susceptible to got highest number of uropathogens and most of the gram positive bacteria were resist to antibiotics that specified to UTI and most commonly given by consultants directly without culturing.

\section{Acknowledgement}

We have many thanks for lecturers and friends who helped us during this research. We would like to thank the Biology Department, Faculty of Science from Soran University and Ashti Hospital to all their support.

\section{References}

1. Gastmeier P, Kampf G, Wischnewski NA, Hauer T, Schulgen G (1998) Prevalence of nosocomial infections in representative German hospitals. J Hospital Infection 38: 37-49.

2. Najar MS, Saldanha CL, Banday KA (2009) Approach to urinary tract infections. Indian J Nephrol 19: 129-139.

3. Khan IU, Mirza IA, Ikram A, Afzal A, Ali S, et al. (2014) Antimicrobial susceptibility pattern of bacteria isolated from patients with urinary tract infection. $J$ Coll Physicians Surg Pak 24: 840-844.

4. Onu GA, Korir SC, Cheruiyot JC, Ratemo OD, Mabeya BM, et al. (2013) Isolation, identification and characterization of urinary tract infections bacteria and the effect of different antibiotics. J Nat Sci Res 3: 150-159.

5. Gonzalez CM, Schaeffer AJ (1999) Treatment of urinary tract infection: What's old, what's new, and what works. World J Urol 17: 372-382.

6. Liang FX, Bosland MC, Huang H, Baptiste S, Deng FM, et al. (2005) Cellular basis of urothelial squamous metaplasia roles of lineage heterogeneity and cell replacement. J Cell Biol 171: 835-844.

7. Foxman B (2003) Epidemiology of urinary tract infections: Incidence, morbidity, and economic costs. Am J Med: 113.

8. Weichhart T, Haidinger M, Hörl WH, Säemann MD (2008) Current concepts of molecular defence mechanisms operative during urinary tract infection. Eur $\mathrm{J}$ Clin Invest 38: 29-38.

9. Williams G, Craig JC (2011) Long $\square$ term antibiotics for preventing recurrent urinary tract infection in children. Cochrane Database Syst Rev.

10. Vasudevan R (2014) Urinary tract infection: An overview of the infection and the associated risk factors. J Micro Exp 1: 8.

11. Balakrishnan, Hill V (2010) Dealing with urinary tract infections. The Pharm J 287: $687-690$

12. Ronald A (2003) The etiology of urinary tract infection: Traditional and emerging pathogens. Dis Mon 49: 71-82.

13. Stamm WE, Hooton TM (1993) Management of urinary tract infections in adults. N Engl J Med 329: 1328-1334

14. Kibret M, Abera B (2014) Prevalence and antibiogram of bacterial isolates from urinary tract infections at dessie health research laboratory, Ethiopia. Asian Pac J Trop Biomed 4: 164-168.

15. Farajnia S, Alikhani MY, Ghotaslou R, Naghili B, Nakhlband A (2009) Causative agents and antimicrobial susceptibilities of urinary tract infections in the northwest of Iran. Int J Infect Dis 13:140-144.

16. Hooton TM (1999) Practice guidelines for urinary tract infection in the era of managed care. Int J Antimicrob Agents 11: 241-245.

17. Schaeffer AJ, Rajan N, Cao Q, Anderson BE, Pruden DL, et al. (2001) Host pathogenesis in urinary tract infections. Infect Dis Clin North Am 17: 245-251.

18. Hooton TM (2000) Pathogenesis of urinary tract infections: An update. J Antimicrob Chemother: 1-7.

19. Andreu A (2005) Pathogenesis of urinary tract infections. Enfermedades infecciosasy microbiologia clinica 23: 15-21.

20. Guglietta A (2017) Recurrent urinary tract infections in women: Risk factors etiology, pathogenesis and prophylaxis. Future Micro 12: 239-246.

21. Foxman B (2010) The epidemiology of urinary tract infection. Nat Rev Urol 7 : $653-660$.

22. Terraf MCL, Tomás MSJ, Rault L, Le Loir Y, Even S, et al. (2017) In vitro effect of vaginal lactobacilli on the growth and adhesion abilities of uropathogenic Escherichia coli. Arch Microbiol 1-8.

23. Tony Mazzulli MD (2012) Diagnosis and management of simple and complicated urinary tract infections (UTIs). Can J Urol 19: 42-48.

24. Kline KA, Lewis AL (2015) Gram-positive uropathogens, polymicrobial urinary tract infection, and the emerging microbiota of the urinary tract. Micro spec: 4 .

25. Kranz R, Weston-Hafer K, Richards E (2006) Identifying unknown bacteria using biochemical and molecular methods. Washington University in Saint Louis.

26. Vickers AA, Chopra I, O'neill AJ (2007) Intrinsic novobiocin resistance in Staphylococcus saprophyticus. Antimicrob Agent Chem 51: 4484-4485. 
Citation: Karzan Mohammed K, Faeza Burhan O and Shahida Nooruldeen Y (2017) Isolation and Identification of Urinary Tract Infectious Bacteria and Exploring their Anti-drug Potential against Some Common Antibiotics. J Microb Biochem Technol 9: 285-289. doi: 10.4172/1948-5948.1000379

27. Boyce JM, Potter-Bynoe G, Chenevert C, King T (1997) Environmental contamination due to methicillin-resistant Staphylococcus aureus possible infection control implications. Infect Control Hosp Epidemiol 18: 622-627.
28. Hoberman A, Wald ER (1997) Urinary tract infections in young febrile children Pediatr Infect Dis J 16:11-17.

29. Koneman EW (1997) Color atlas and textbook of diagnostic microbiology Lippincott-Raven Publishers. 